\title{
P287
}

\section{Estimation of Stress Induced Azimuthal Anisotropy - AVO Modeling}

\author{
M. Brajanovski* (Curtin University of Technology), B. Gurevich (Curtin \\ University of Technology), T. Lokajicek (Institute of Geology, AS CR), D. \\ Nadri (CSIRO), A. Bona (Curtin University of Technology) \& G. Duncan \\ (BHPBilliton)
}

\section{SUMMARY}

The analysis of rock anisotropy in terms of seismic velocities and within the context of rock-physics (BiotGassmann theory of poroelasticity) provides important information for the evaluation of the stress state (tensors) of rocks, detection of the directions of formation weaknesses, helps in the estimation of overall permeability and failure prediction. Understanding the influence of stress and pore pressure on seismic velocities is important for 4-D reflection seismic interpretation, AVO analysis and reservoir modeling. Laboratory measurements were carried out on spherical shale samples from the overburden under confining stress up to $400 \mathrm{MPa}$, by means of ultrasonic soundings in 132 independent directions. Such an approach enables the estimation of 3-D elastic anisotropy. Assuming VTI symmetry approximation, from the measured velocities the stiffness tensor was inverted. Since the sandstones were partly unconsolidated, it was not possible to take ultrasonic measurements . To overcome this, we developed a method for stress induced azimuthal anisotropy estimation using only cross-dipole logging data. These results give the possibility for anisotropic correction in AVO analysis. 


\section{Introduction}

Unusual seismic wave anisotropy was observed in both marine and cross-dipole logging data sets. The existing data show travel-time anisotropy in the scale of kilometers (surface seismic) down to the borehole logs in the scale of meters (strong shear wave splitting). In addition, laboratory measurements confirm anisotropy at the scale of centimeters. The main reason for this anisotropy was asymmetric horizontal stress. Anisotropy affects AVO and must be taken into account for correct interpretation. In order to understand stress induced anisotropy we built a new rock physics model, which allows us to estimate the HTI elasticity tensor of a sandstone reservoir from log data only. Also, using acoustic laboratory measurements carried out on spherical shale samples we estimate the VTI tensor representing the overburden. Finally, knowing previous tensors we demonstrate the effect of anisotropy on AVO.

\section{Experimental evidence of anisotropy}

The package of interest consists of a thin (up to 15 meters) sandstone reservoir, very poorly consolidated, and a thick shale overburden. Existing marine data show significant (up to 5\%) azimuthal P-wave travel-time anisotropy in the overburden (Hung et al. 2006). The sandstone reservoir shows much greater azimuthal anisotropy. Figure 1 shows that the relative difference between fast and slow shear waves (measured by the cross-dipole logging tool) goes up to $20 \%$ in the sandstone reservoir and less than $3-4 \%$ in the shale.

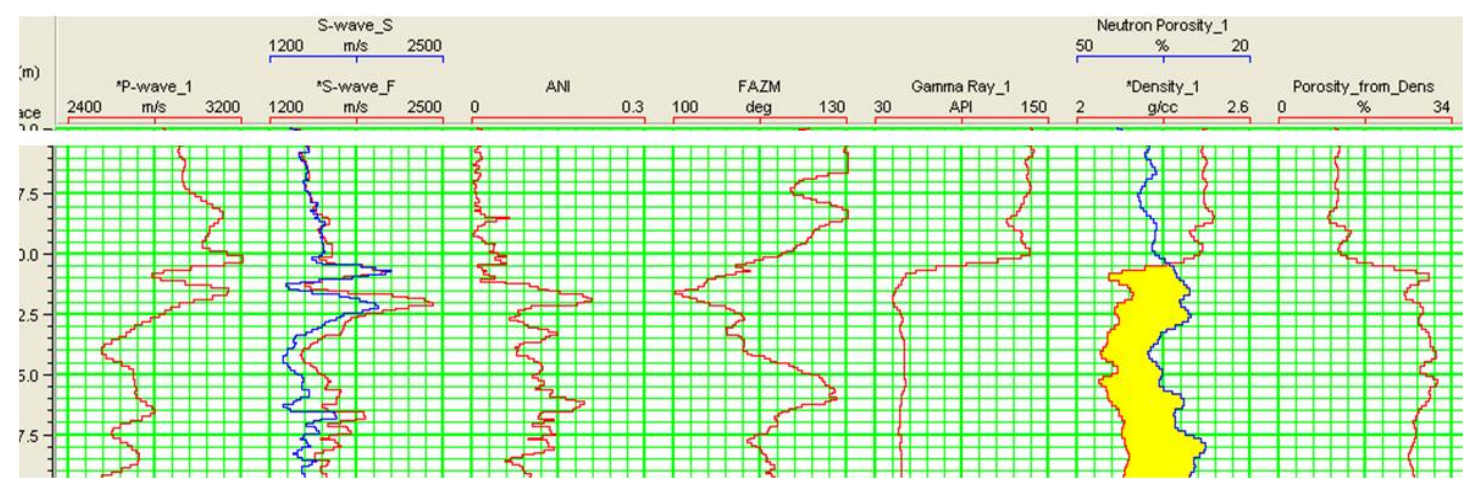

Figure 1: A portion of the cross-dipole well data showing substantial shear wave velocity anisotropy in the sand (yellow fill) and low anisotropy in the shale.

From the fact that same stress field produces very different degrees of shear wave anisotropy in sand and shale we conclude that shale behaves to some extent as a plastic material (small shear wave stress-induced anisotropy) for slow processes (isothermal deformation).

\section{Spherical sample measurements and tensor estimation for shale}

To get an unbiased estimation of shale anisotropy the measurements were performed on a spherical sample. A core sample was cut and polished to obtain a sphere $50 \mathrm{~mm}$ in diameter. The sample was placed in a pressure chamber to measure the ultrasonic velocities in a range of confining pressures up to 400MPa. Travel-times were measured over the spherical sample every $15^{\circ}$ in azimuthal and polar directions using $2 \mathrm{MHz}$ transducers. This acquisition pattern produced $132 \mathrm{P}$-wave records, from which velocities were calculated and interpolated over the whole surface of the sphere as shown in Figure 2 (left). From Figure 2 (left) we see approximately $2-3 \%$ relative velocity difference in the bedding plane, which may contribute to overall azimuthal travel-time anisotropy observed by Hung et al. (2006).

From P-wave travel times measured at 40MPa (in situ stress) we find the symmetry axis. We transform the ray velocities from the measurement coordinate system to the symmetry axis coordinate system. Then, assuming VTI symmetry, we estimated the elasticity tensor using the Simulating Annealing followed by quasi Newton algorithm (Nadri et al., 2009). 


\section{Amsterdam 'og}

An additional interesting phenomenon can be seen from these measurements. Figure 2 (right) shows an azimuthally dependent amplitude response in the bedding plane of the shale. This is a surprising result. It can be understood as a consequence of asymmetric horizontal stress. The shear wave splitting induced by stress in shale is negligible but azimuthally dependent amplitude response (quality factor) is certainly not.
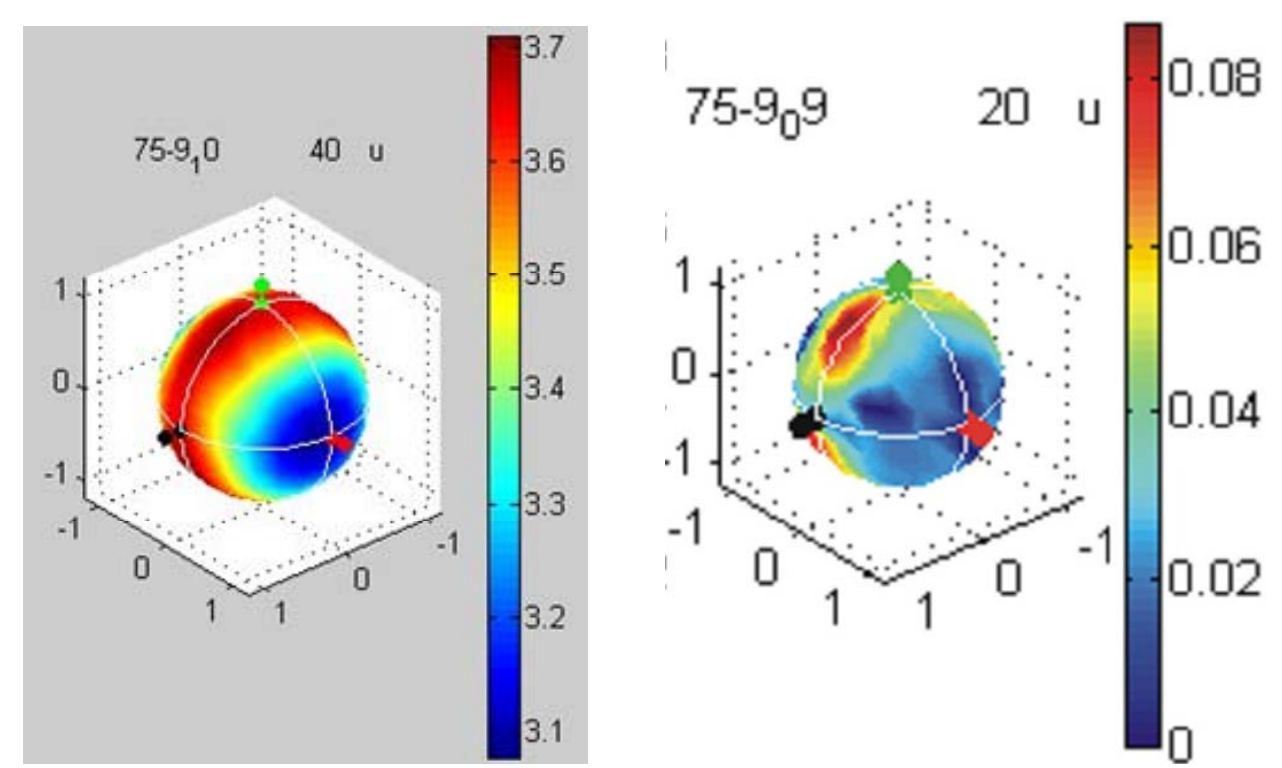

Figure 2: P-wave velocity (left) and amplitude response (right). The red dot indicates vertical ( $z \equiv x_{3}$ ) axis

\section{Stress induced transversely isotropic (SITI) tensor estimation for sandstone reservoir}

In order to invert the elastic tensor of shale we used laboratory measurements carried out on the spherical samples. For this particular borehole, measurements of core samples of sand cannot be done because the rock is almost unconsolidated and it is impossible to cut a sample. Instead, we developed a method for estimation of HTI (SITI) elasticity tensor of pre-stressed sandstone based on cross-dipole logging data.

Asymmetric horizontal stress may cause rock to fracture or to have a different "elastic grain contact quality" in different directions, with such deformations commonly aligned with the dominant horizontal stress direction. The azimuth of such fractures or planes of weaknesses formed by contact voids depends on the azimuth of the dominant stress direction in the horizontal plane. We use the term "plane of weakness" rather than fracture because it describes softness of the medium in general, independently is it made up of tens of metre long fractures or thousands of micrometer small contact voids, which can sum up to a "plane" in the presence of external laterally anisotropic stress. Even without macro-cracks or fractures, rock matrix has the potential to become anisotropic under external stress because of the existence of two kinds of pores. For stiff pores (aspect ratio goes to 1) the relative change of volume is also small, but for compliant pores (aspect ratio $~ 0.001$ ), like grain contact voids, this relative change of pore volume caused by the "negative" external stress could be huge and they can open, causing weaker contact and consequently decrease Young's modulus of dry rock matrix (Shapiro, 2003).

Even though the elastic tensor of sandstone reservoirs is orthorhombic in general (three different principal stress directions), we will use HTI symmetry approximation, assuming that vertical and maximal horizontal traction still keep contact voids and micro-cracks closed, while minimal horizontal traction acts effectively as a stretching force producing a TI effective medium with $x_{1}$ being axis of rotational symmetry. From inequality of $c_{55}$ and $c_{44}$ (cross-dipole logs) at any frequency it is obvious that differently polarized shear waves will have different velocities. 


\section{Amsterdam 'og}

In general, three different principal stresses will cause three different excess compliances originating from the contact voids. Schoenberg (1988) and Brajanovski et al., (2005) showed that the contribution of such "grain contact softness" to the isotropic background compliance can be described by introducing "fracture excess compliance". HTI (SITI) approximation means that $Z_{N 2} \approx Z_{N 3}$ and $Z_{T 2} \approx Z_{T 3}$. Now we can subtract $Z_{N 2}$ and $Z_{T 2}+Z_{T 3}$ (taking into account both directions of tangential excess compliances from grain contacts) from the fracture compliance matrix and get "effective weakness" matrix

$$
s_{f}=\left(\begin{array}{cccccc}
Z_{N 1} & 0 & 0 & 0 & 0 & 0 \\
0 & Z_{N 2} & 0 & 0 & 0 & 0 \\
0 & 0 & Z_{N 3} & 0 & 0 & 0 \\
0 & 0 & 0 & Z_{T 2}+Z_{T 3} & 0 & 0 \\
0 & 0 & 0 & 0 & Z_{T 1}+Z_{T 3} & 0 \\
0 & 0 & 0 & 0 & 0 & Z_{T 1}+Z_{T 2}
\end{array}\right) \equiv\left(\begin{array}{cccccc}
Z_{N e f} & 0 & 0 & 0 & 0 & 0 \\
0 & 0 & 0 & 0 & 0 & 0 \\
0 & 0 & 0 & 0 & 0 & 0 \\
0 & 0 & 0 & 0 & 0 & 0 \\
0 & 0 & 0 & 0 & Z_{T e f} & 0 \\
0 & 0 & 0 & 0 & 0 & Z_{T e f}
\end{array}\right) .
$$

Also, we anticipate the relation between normal and tangential excess compliance to be $Z_{N}=r Z_{T}$ (because of simplicity we do not use "ef" in index) and in that case we need only three parameters $\lambda_{b}, \mu_{b}, Z_{N}$ (index " $b$ " means background) in order to estimate stiffness tensor for TI symmetry. Rasolofosaon (1998) showed that application of anisotropic stress to an isotropic rock results in ellipsoidal anisotropy. This means that for dry rock Thomsen's anisotropy parameters $\varepsilon$ and $\delta$ are equal, which is equivalent to $Z_{N}=Z_{T}$. Based on the papers of Gurevich (2003) and Brajanovski et al. (2005) we developed a method, which gives the complete solution for stress induced HTI (SITI) anisotropy using cross-dipole data only.

\section{AVO modeling}

At an interface of two homogenous transverse isotropic (TI) media with different symmetry axis directions, vertically transverse isotropic (VTI) layer over a horizontally transverse isotropic (HTI) layer, the reflection coefficient for $P \rightarrow P$ mode can be computed using Schoenberg-Protázio equations (1992). Based on previously estimated Thomsen's parameters in the overburden shale and the sandstone reservoir we created blocky logs for $\varepsilon, \delta$, and $\gamma$. We used the sonic P-wave $\log$ as the vertical $\mathrm{P}$-wave velocity and the slow mode of shear wave as the vertical shear wave velocity. To understand the azimuthal variations of AVO we have computed the reflection coefficients for different survey azimuths with respect to the maximal stress direction (isotropy plane). Figure 3 shows the reflection coefficients at the interface of the overburden shale and sandstone for azimuths 0 (maximal stress direction), $30^{\circ}, 45^{\circ}, 60^{\circ}$ and $90^{\circ}$ (minimal stress direction) for different offsets. These offsets correspond to incidence angles in a range $0-60^{\circ}$. There is a significant change in reflectivity for large offsets. To compare with the isotropic case, we have also computed the isotropic reflectivity at the same interface. As can be seen there is a substantial difference. Modeling shows that the effect of anisotropy has to be taken into account in AVO analysis.

\section{Conclusion}

We investigated the influence of asymmetric horizontal stress on AVO seismic signatures. We targeted the thin sandstone reservoir below the thick shale overburden. Laboratory measurements were carried out on spherical shale samples from the overburden under confining stress up to 400MPa, by means of ultrasonic soundings in 132 independent directions. Such an approach enables 3D P-wave elastic anisotropy to be estimated. From the measured velocities, the stiffness tensor was inverted, assuming VTI symmetry approximation. Since the sandstones were partly unconsolidated, it was not possible to take 


\section{Amsterdam 'og}

ultrasonic measurements. In order to overcome this problem we developed a method for estimating stress induced HTI anisotropy using only cross-dipole logging data, and using the results to make an anisotropic correction in AVO analysis.

\section{Acknowledgments}

We are grateful to BHP Billiton Petroleum and the sponsors of Curtin Reservoir Geophysics Consortium for financial support of this project. We thank Milovan Urosevic for useful discussions.

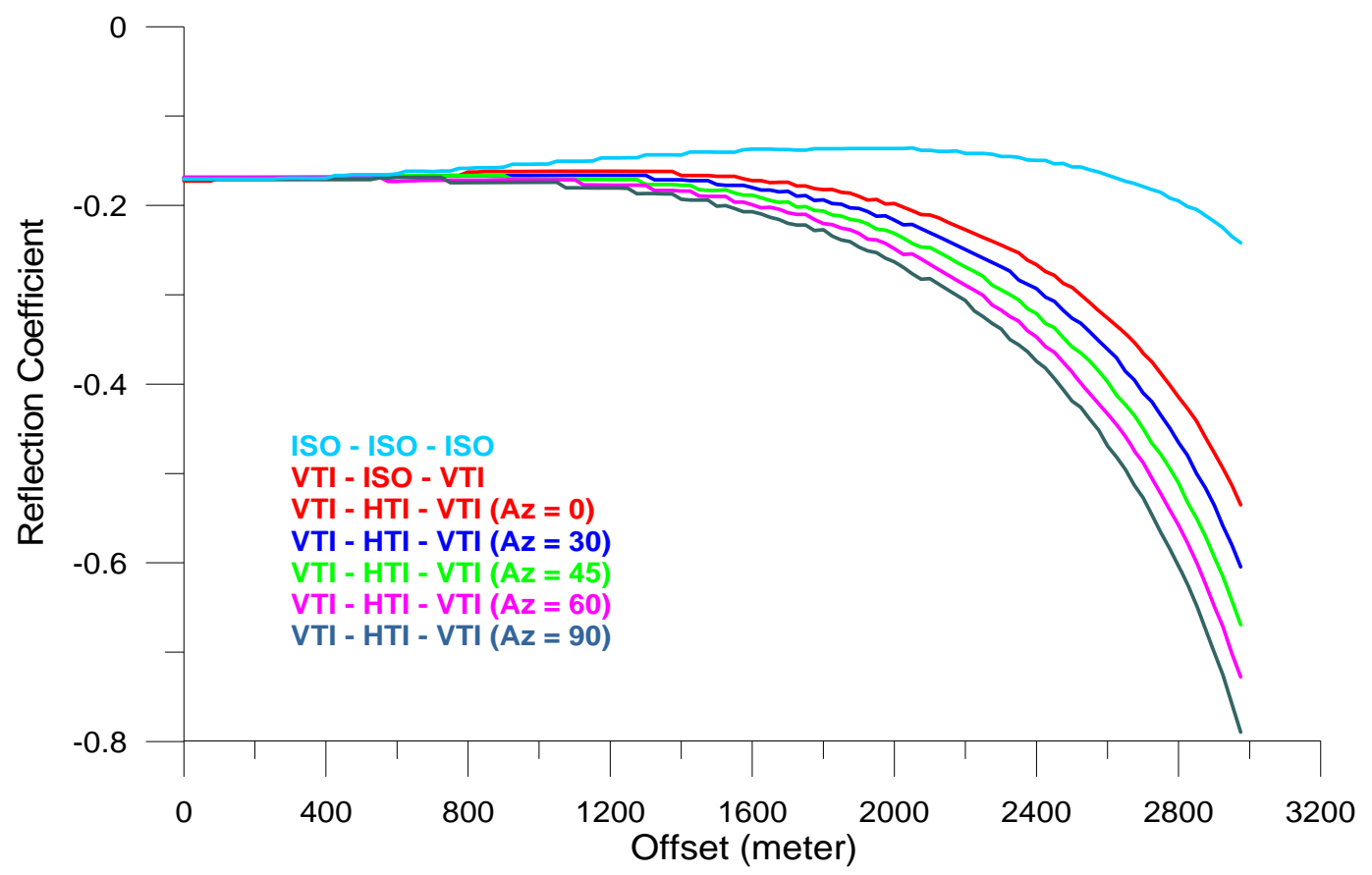

Figure 3: Reflection coefficient versus offset for different azimths and different symmetry classes.

\section{References}

Brajanovski, M., B. Gurevich, and M. Schoenberg, 2005. A model for P-wave attenuation and dispersion in a porous medium permeated by aligned fractures, Geophys. J. Int., 163:372-384, doi:10.1111/j.1365-246X.2005.02722.x.

Gurevich, B., 2003. Elastic properties of saturated porous rocks with aligned fractures, Journal of Applied Geophysics, 54, 203-218.

Hung, B., F. M. Zhang, J. Sun, M. Stanley, and Osadchuk, 2006. An Automated 3D Method for Azimuthal Anisotropy Analysis in Marine Seismic Data, 68th EAGE Annual Meeting, Vienna, H035.

Nadri, D., A. Bona, and M. Brajanovski, 2009. Elasticity tensor inversion from spherical sample measurements, Submitted to 71th EAGE Annual Meeting, Amsterdam.

Rasolofosaon, P., 1998. Stress-Induced seismic anisotropy revisited, Revue De L'Institut Francais Du Petrole, 53, 5, 679-692.

Shapiro, S. A. 2003. Elastic piezosensitivity of porous and fractured rocks, Geophysics, 68. 482-486.

Schoenberg, M. and J. Douma, 1988. Elastic-wave propagation in media with parallel fractures and aligned cracks, Geophysical Prospecting, 36, 571-590.

Shoenberg, M., and J. Protázio, 1992. Zoeppritz rationalized and generalized to anisotropy, Journal of Seismic Exploration, 1, 125-144. 